\title{
Retaining mTeSR1 Medium during Hepatic Differentiation Facilitates Hepatocyte-Like Cell Survival by Decreasing Apoptosis
}

\author{
Jian Hou ${ }^{\mathrm{a}}$ Yan Long ${ }^{\mathrm{a}}$ Bo Hu $\mathrm{Hu}^{\mathrm{b}}$ Shaojie Huang ${ }^{\mathrm{a}, \mathrm{c}}$ Guangtao Xu $\mathrm{Ku}^{\mathrm{b}}$ Tesheng Gao \\ Fenfang $\mathrm{Wu}^{\mathrm{d}}$ Yinxiong $\mathrm{Li}^{\mathrm{d}}$ Zhongkai Wu${ }^{\mathrm{a}}$ \\ aDepartment of Cardiac Surgery, The First Affiliated Hospital of Sun Yat-sen University, Guangzhou, \\ ${ }^{b}$ Department of Molecular Pathology, Jiaxing Hospital of Traditional Chinese Medicine, Jiaxing \\ University, Jiaxing, 'NHC Key Laboratory of Assisted Circulation, Sun Yat-sen University, Guangzhou, \\ Institute of Public Health, Guangzhou Institutes of Biomedicine and Health, Chinese Academy of \\ Sciences, Guangzhou, China
}

\section{Key Words}

Stem cell $\bullet$ IPS cell $\bullet$ Hepatocyte-like cell $\bullet \mathrm{mTeSR} 1$ medium $\bullet$ Apoptosis $\bullet$ Hepatic differentiation

\begin{abstract}
Background/Aims: Hepatocyte-like cells derived from human pluripotent stem cells could be an important cell source for hepatocyte transplantation. The present study investigated the effect of retaining mTeSR 1 medium during hepatic differentiation on hepatocyte-like cells in vitro. Methods: Human embryonic stem cell line $\mathrm{H} 1$ were treated with activin $\mathrm{A}$ and bone morphogenetic protein 4 (BMP4) for definitive endoderm (DE) cell induction and subsequently treated with BMP2 and fibroblast growth factor 4 (FGF4) for early hepatic cell induction. Hepatocyte growth factor (HGF) and fibroblast growth factor (KGF) were added for early hepatic cell expansion and then mixed with oncostatin-M for maturation. During DE induction, $0 \%, 25 \%, 50 \%$ and $75 \%$ concentrations of mTeSR 1 medium were separately added for early hepatic induction and expansion. For optimization, the expression levels of SRY-related HMG-box 17 (SOX17) and forkhead box A2 (FOXA2) at day 4, alpha fetoprotein (AFP) and hepatocyte nuclear factor $4 \alpha$ (HNF4 $\alpha$ ) at day 15, and albumin (ALB) at day 25 were quantified in differentiated cells by $q R T-P C R$. The ALB-positive cell proportion was measured by flow cytometry. Functional tests including ALB secretion and indocyanine green (ICG) angiography uptake and release by ELISA, urea production by urea assay kit, and glycogen storage ability by periodic acid Schiff reaction (PAS) staining were performed in the differentiated cells. The induced pluripotent stem (iPS) cells were used to examine whether the optimized method was suitable for differentiating iPS cells. DE and hepatic markers were detected by immunostaining, and functional testing was performed as described above. Flow cytometry with an Annexin V-FITC apoptosis detection kit and fluorescence microscopy with Hoechst 33258 were used to analyze apoptosis in differentiated cells derived from J. Hou., Y. Long, and B. Hu contributed equally to this work.

Dr. Zhongkai Wu, Dr. Yinxiong Li and Dr. Fenfang Wu

The First Affiliated Hospital of Sun Yat-sen University; Guangzhou Institutes of Biomedicine and Health Chinese Academy of Sciences, 58 Zhongshan Second Rd., Guangzhou 510080, GD (China) E-Mail wuzhk@mail.sysu.edu.cn; liyinxionggibh@163.com; wufenfang19@126.com
\end{abstract}

KARGER 
H1 cells. Results: All differentiated cells with retention of $0 \%, 25 \%, 50 \%$ and $75 \%$ mTeSR1 expressed SOX17, FOXA2, AFP, HNF4 $\alpha$, and ALB, while higher expression levels were observed in differentiated cells in the $0 \%$ and $25 \%$ groups. The flow cytometry results showed that the proportion of ALB-positive differentiated cells derived from $\mathrm{H} 1$ cells was higher in the $25 \%$ mTeSR1 group than in other groups. However, no significant difference in ALB secretion, urea production, ICG uptake and release and glycogen storage ability was detected between the $25 \%$ and $0 \%$ groups. The iPS cells could differentiate into hepatocyte-like cells with $25 \%$ mTeSR1 retention. The apoptosis ratio of differentiated cells was lower in the 25\% mTeSR 1 group than in the 0\% mTeSR1 group. Conclusion: Retaining 25\% mTeSR1 medium during hepatic differentiation has been proposed to increase the percentage of ALB-positive cells and cell survival by decreasing cell apoptosis.

(C) 2018 The Author(s)

Published by S. Karger AG, Basel

\section{Introduction}

Liver dysfunction is one of the most important global health problems, and liver transplantation is the only successful therapy for end-stage liver failure $[1,2]$. However, the availability of human liver donors is limited. Hepatocyte transplantation, as an alternative to organ transplantation, restores damaged liver function and provides a potential way to treat a wide range of liver diseases [3]. Nevertheless, the application of primary human hepatocytes has been hindered by a lack of cellular growth, loss of function and dedifferentiation in vitro [4]. Thus, the generation of functional hepatocytes from other unlimited sources would be greatly beneficial.

Human embryonic stem (ES) cells (hESCs) are characterized by pluripotency and the capacity for unlimited self-renewal. In addition, growth factors or microRNAs have been reported to contribute to hepatocyte differentiation [5, 6]. Moreover, several studies found that hepatocyte-like cells could be derived from hESCs and that pluripotent stem cells could serve as a potentially inexhaustible source of cells for replacement therapies [7-9]. Thus, in the present study, we focused on the effect of different mTeSR1 culture conditions on the differentiation of human ES cells and induced pluripotent stem cells (iPS) cells to optimize robust and reliable methods of hepatic differentiation in vitro.

\section{Materials and Methods}

\section{Cell culture and differentiation}

The human ESC line H1 was obtained from WiCell Research Institute (Madison, WI). The passage number of the $\mathrm{H} 1$ cells and iPS cells used ranged from 44 to $60[10,11]$. The H1 hESCs were plated on Matrigel (BD Biosciences, San Jose, CA)-coated plates and maintained in mTeSR1 medium (StemCell Tech., Vancouver, BC, Canada). For endoderm induction, the H1 cells were incubated for 2 days with RPMI 1640 (Invitrogen) plus $0 \%, 25 \%, 50 \%$ or $75 \%$ mTeSR 1 medium (StemCell), supplemented with $100 \mathrm{ng} / \mathrm{mL}$ activin A and $20 \mathrm{ng} /$ mL BMP4 (both from Peprotech, Rocky Hill, NJ) and then with $100 \mathrm{ng} / \mathrm{mL}$ activin A for an additional 2 days. Following treatment with activin A, the differentiated H1 cells were cultured in RPMI 1640 with $30 \mathrm{ng} / \mathrm{mL}$ FGF4 and $20 \mathrm{ng} / \mathrm{mL}$ BMP2 (both from Peprotech) for 5 days. Next, the differentiated cells were incubated in RPMI 1640 plus $0 \%, 25 \%, 50 \%$ or $75 \%$ mTeSR 1 medium (StemCell) with $20 \mathrm{ng} / \mathrm{mL}$ HGF and $20 \mathrm{ng} / \mathrm{mL} \mathrm{KGF}$ (both from Peprotech) for 12 days. Subsequently, the differentiated cells were treated with high-glucose DMEM (Thermo Fisher Scientific, Hampton, NH) with ascorbic acid (1:1000), bovine serum albumin-fatty acid free (1:500), transferrin (1:1000), insulin (1:1000), gentamicin/amphotericin-B (1:1000) (all from Lonza Sales Ltd, Basel, Switzerland), and $10 \mathrm{ng} / \mathrm{mL}$ oncostatin-M (OSM; R\&D, Minneapolis, MN, USA) for another 10 days. The iPS cell line culture was the same as the method mentioned above. 


\section{Cellular Physiology Cell Physiol Biochem 2018;51:1533-1543 and Biochemistry \begin{tabular}{l|l} 
DOI: 10.1159/000495644 2018 The Author(s). Published by S. Karger AG, Basel \\
Publishedon
\end{tabular}

\section{RNA isolation and $q R T-P C R$}

Total RNA was isolated from the cultured cells using TRIzol Reagent (Thermo Fisher Scientific) and was reverse transcribed by a PrimeScript RT reagent kit with gDNA Eraser (Takara, Dalian, China) according to the manufacturer's protocol. Polymerase chain reaction (PCR) was performed with GoTaq Green MasterMix (Promega, Madison, WI, USA). Primers were designed as previously described [12] and are listed in Table 1.

\section{Immunofluorescence assay}

Cells were fixed with 4\% paraformaldehyde for 15 minutes and then blocked and permeabilized with $1 \%$ BSA and $0.2 \%$ Triton X-100 in phosphate-buffered saline (PBS) at room temperature for $1 \mathrm{~h}$. Next, the cells were incubated with primary antibody overnight at $4{ }^{\circ} \mathrm{C}$. The primary antibodies used were goat antiSOX17 (R\&D, AF1924), rabbit anti-FOXA2 (R\&D, AF2400), mouse anti-AFP (Santa Cruz Biotechnology, CA, sc-130302), mouse anti-HNF4 $\alpha$ (Genetex, San Antonio, TX, GTX62347), mouse anti-ALB (R\&D, MAB1455), and mouse anti-CK19 (Genetex, GTX112666), and all were diluted at 1:200. After five washes with PBS, the cells were incubated with FITC-conjugated and TRITC-conjugated secondary antibodies diluted at 1:500 for $1 \mathrm{~h}$ at room temperature. Finally, the cells were incubated with $1 \mu \mathrm{g} / \mathrm{mL}$ 4, 6-diamidino-2-phenylindole (Sigma, St. Louis, MO, USA) to stain the cell nuclei. Additionally, the appropriate isotype antibody and normal serum from the species corresponding to the primary antibody were used as negative controls. Cells were analyzed by a confocal fluorescence microscope (Zeiss 710 NLO, Carl Zeiss Microscopy, LLC, Thornwood, NY, USA).

\section{ELISA for $A L B$}

The content of human albumin in the supernatant was determined by a Human Albumin ELISA Kit (Koma Biotech, Seoul, Korea) according to the manufacturer's instructions. Cells were trypsinized and counted with a hemocytometer.

\section{Urea assay}

Urea production was detected by a QuantiChrom Urea assay kit (Bioassay Systems, Brussels, Belgium). Cells were trypsinized and counted with a hemocytometer. The supernatant samples were stored at $-20^{\circ} \mathrm{C}$, and the assay was performed according to the manufacturer's instructions.

\section{PAS staining}

Cells in culture dishes were fixed in $70 \%$ ethyl alcohol, and staining was performed according to the manufacturer's instructions (Cat. 24200, Polysciences).

Cellular uptake and release of ICG

ICG (TCI, I0535) was dissolved in DMSO to make a stock solution at $50 \mathrm{mg} / \mathrm{mL}$ and then freshly diluted in culture medium to $1 \mathrm{mg} / \mathrm{mL}$. After incubation of cells with ICG for $30 \mathrm{~min}$ at $37^{\circ} \mathrm{C}$, the medium with ICG was discarded, cells were washed with PBS, and cellular uptake of ICG was examined by microscopy. The cells were then returned to the culture medium and incubated for $6 \mathrm{~h}$ to release the cellular ICG stain.

\section{Analyses of apoptotic cells by flow cytometry}

Annexin V-FITC apoptosis detection was reported previously [13]. An Annexin V-FITC Kit (Beyotime, China) was used to analyze the apoptotic cells. Briefly, cells were collected and incubated with Annexin V-FITC and PI in the provided binding buffer for $30 \mathrm{~min}$ in the dark at $4^{\circ} \mathrm{C}$. They were then subjected to flow cytometry analysis at an emission wavelength of $488 \mathrm{~nm}$.

\section{Hoechst staining}

Hoechst staining was performed using an Apoptosis-Hoechst Staining kit (Beyotime) according to the manufacturer's instructions. In brief, the cells were seeded in 24-well plates and fixed with $4 \%$ paraformaldehyde, stained with Hoechst 33528 and then visualized under a fluorescence microscope (Zeiss 710 NLO).

Table 1. Primers for qRT-PCR

\begin{tabular}{lc}
\hline Gene & Primers \\
\hline \multirow{2}{*}{ SoX17 } & F: GAACGCTTTCATGGTGTGGGCTAAG \\
& R: CCACGACTTGCCCAGCATCTTGC \\
FOXA2 & F: GGTGATGCACTACCCCGGCTACG \\
& R: CAGGGGCGAGGCGTCCAGG \\
AFP & F: GCGAAGCTGCGGCTCTTCCAG \\
& R: GCTGTGGCTGCCATTTTTCTGGTGAT \\
HNF4a & F: AGAAGGCACCAACCTCAACGC \\
& R: GGGCAGGCTGCTGTCCTCATA \\
ALB & F: GGTGTTGATGCCTTTGCTCAGTATC \\
& R: CCCTTCATCCCGAAGTTCATCGA \\
GAPDH & F: ATCCTGGGCTACACTGAGCACC \\
& R: AAGTGGTCGTTGAGGGCAATG \\
\hline
\end{tabular}




\section{Cellular Physiology Cell Physiol Biochem 2018;51:1533-1543

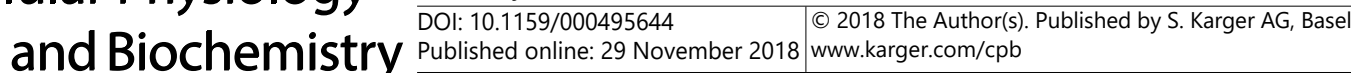 \\ Hou et al.: mTeSR1 Medium during Hepatic Differentiation}

Statistical analysis

Data are expressed as the mean \pm standard error of the mean (SEM). A Microsoft Excel 2016 database and GraphPad Prism 5 software (GraphPad Software Inc., La Jolla, California) were used to record and analyze all the data. One-way analysis of variance (ANOVA) with a post hoc test or Student's $t$-test were used for data analysis. Differences with a p-value of $<0.05$ were considered statistically significant.

\section{Results}

Retaining a 25\% concentration of mTeSR1 medium could increase the proportion of ALBpositive differentiated cells derived from $\mathrm{H} 1 \mathrm{cells}$

Here, we developed a hepatic differentiation method for human pluripotent stem cells, as shown in Fig. 1A. The differentiation protocol was composed of four stages. In the first stage, activin A and BMP4 synergically induced endoderm differentiation. Then, FGF4 and BMP2 were used to induce hepatic specification from endoderm cells, and KGF and HGF were used to expand the early hepatic cells. During these three stages, different concentrations of mTeSR1 medium were retained. In the final stage, the mTeSR 1 medium was removed, and OSM was used to facilitate early hepatic cell maturation. Fig. 1B shows the cell morphology changes with time during the differentiation of $\mathrm{H} 1$ cells treated with $0 \%, 25 \%, 50 \%$ or $75 \%$ mTeSR1. During differentiation, more dead cells were observed in the group without mTeSR1 than in the other groups (data not shown). At day 4, the cells in the four groups displayed endoderm cell morphology. At days 15 and 21, the group treated with $25 \%$ mTeSR 1 displayed better cell morphology of early hepatic cells and mature hepatocytes, respectively, than the other groups (Fig. 1B).

Fig. 1. Generation of hepatocyte-like cells derived from H1 cells. Note: (A) The schema of the stepwise differentiation protocol. Cells were treated with 100 $\mathrm{ng} / \mathrm{mL}$ activin A and $20 \mathrm{ng} / \mathrm{mL}$ BMP4 for 2 days and then with $100 \mathrm{ng} / \mathrm{mL}$ activin A for 2 days to induce the formation of definitive endoderm. Then, cells were treated with $20 \mathrm{ng} / \mathrm{mL}$ BMP2 and $30 \mathrm{ng} /$ mL FGF4 for 5 days to induce early

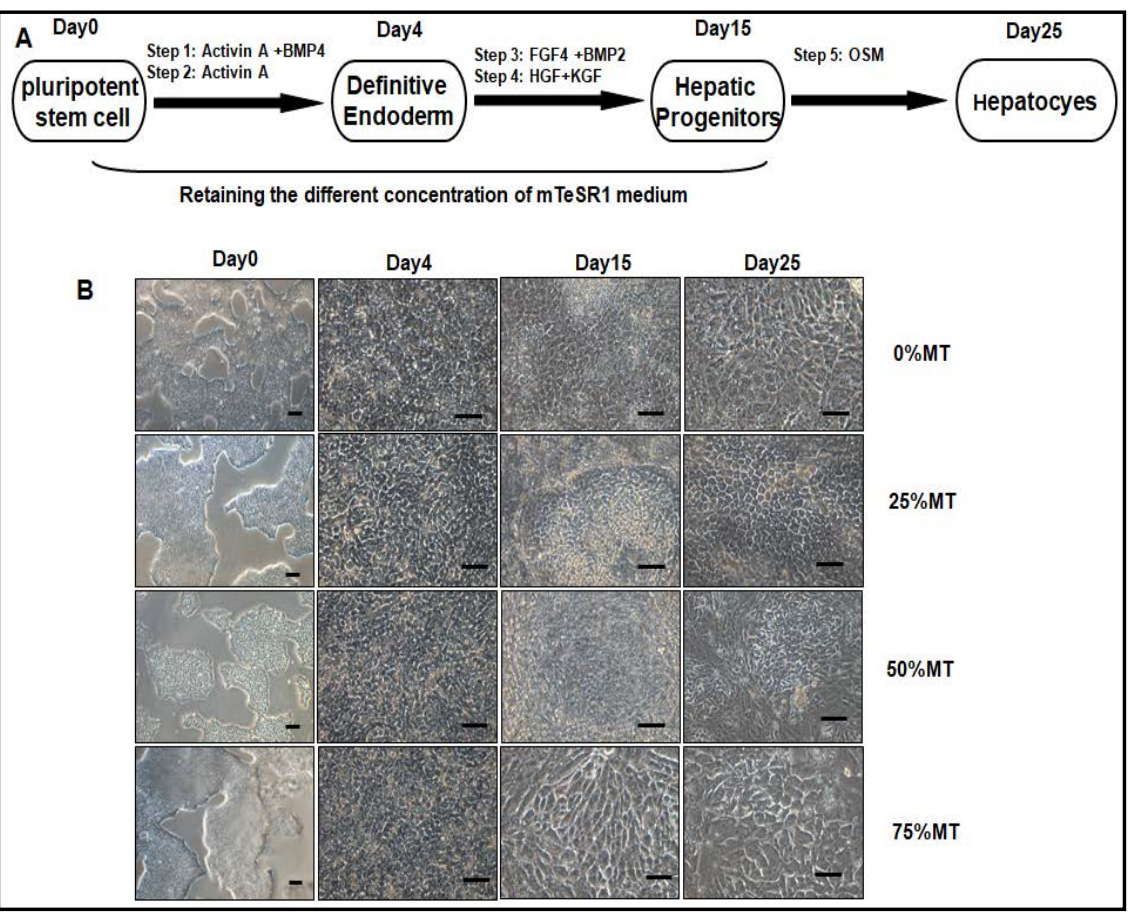
hepatic cells and with $20 \mathrm{ng} / \mathrm{mL} \mathrm{HGF}$ and $20 \mathrm{ng} / \mathrm{mL}$ KGF for 7 days to expand them. During the differentiation protocol above, RPMI 1640 medium plus a $0 \%, 25 \%, 50 \%$ or $75 \%$ concentration of mTeSR1 medium was used. In the mature stage, high-glucose DMEM with $10 \mathrm{ng} / \mathrm{mL}$ oncostatin-M was used for 10 days. (B) The cells on days $0,4,15$ and 25 in bright-field microscopy. Scale bar, $100 \mu \mathrm{m}$. 


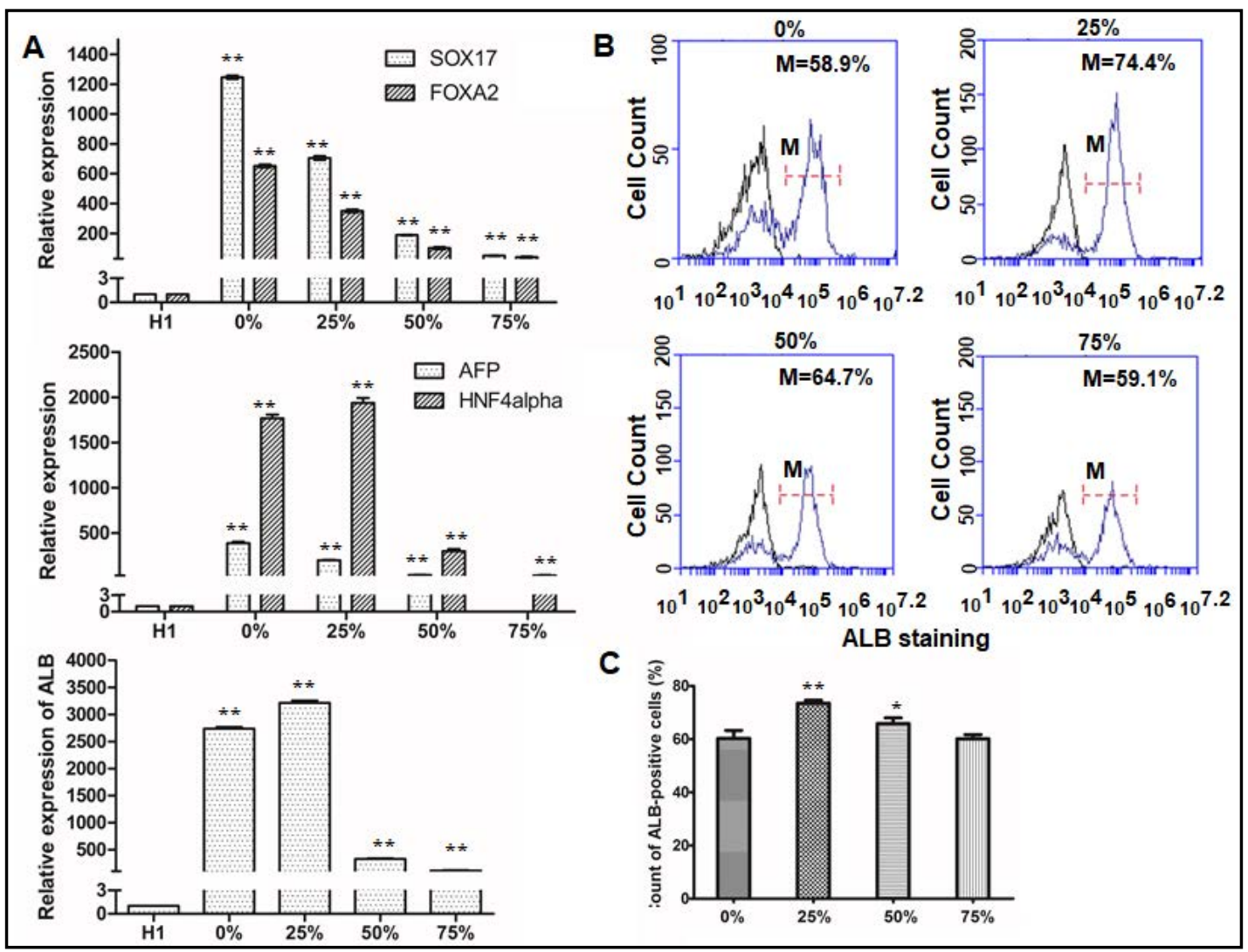

Fig. 2. Characterization of differentiated cells derived from $\mathrm{H} 1$ cells with the indicated markers. Note: (A) The expression of SOX17 and FOXA2 at day 4, AFP and HNF4 $\alpha$ at day 15 and ALB at day 25 were detected in differentiated cells by qRT-PCR. The relative expression levels were normalized to an internal control. The data are expressed as the mean $\pm \mathrm{SD}$. ${ }^{* *} \mathrm{p}=0.000$ vs. H1 cell line. (B) Representative flow cytometry profile and $(\mathrm{C})$ the average number of albumin-positive H1-derived hepatocytes in two independent differentiation experiments. ${ }^{*} \mathrm{p}<0.05,{ }^{* *} \mathrm{p}=0.000$ vs. control group $(0 \%)$.

To further confirm hepatic differentiation, real-time PCR was used to detect markers of endoderm cells, hepatic precursor cells, and mature hepatocytes (Fig. 2A). The differentiated cells in all the groups showed high expression levels of endoderm markers SOX17 $0 \%$ : $1246.036 \pm 11.300,25 \%$ : $705.692 \pm 13.106,50 \%: 189.032 \pm 2.141,75 \%: 50.907 \pm 2.102$, H1 cells normalized as 1 ) and FOXA2 (0\%: $651.779 \pm 10.388,25 \%: 350.850 \pm 10.049,50 \%$ : $101.651 \pm 8.112,75 \%: 40.636 \pm 4.150$, H1 cells normalized as 1 ) at day 4 , hepatic precursor markers AFP (0\%: $387.644 \pm 17.295,25 \%$ : $198.390 \pm 5.310,50 \%$ : $35.623 \pm 3.272,75 \%$ : $0 \pm 0$, H1 cells normalized as 1$)$ and HNF4 $\alpha(0 \%: 1766.782 \pm 41.985,25 \%: 1937.499 \pm$ 54.023, 50\%: $298.390 \pm 24.173,75 \%$ : $31.779 \pm 5.0380$, H1 cells normalized as 1 ) at day 15 , and mature hepatocyte marker ALB (0\%: $2737.499 \pm 31.250,25 \%: 3212.072 \pm 45.122$, 50\%: $328.221 \pm 13.114,75 \%: 114.615 \pm 5.297$, H1 cells normalized as 1 ) at day 21 . These results indicated that hepatocyte-like cells could be obtained from all the groups treated with or without mTeSR1 medium, although the efficiency might be different. To detect the differentiation efficiencies of the four groups, the proportions of ALB-positive cells were analyzed using flow cytometry (Fig. 2B, 2C). Fig. 2B shows that the group with $25 \%$ mTeSR1 medium displayed a higher percentage of ALB-positive cells than the other groups $10 \%$ : 58.9\%; 25\%: 74.4\%; 50\%: 64.7\%; 75\%: 59.1\%). Further analysis of replicates confirmed the results above (Fig. 2C) (0\%: 60.24\% \pm 3.01\%; 25\%: 73.47\% $\pm 1.15 \% ; 50 \%: 65.82 \% \pm$ $2.18 \%$; $75 \%: 60.12 \% \pm 1.56 \%$ ). These results suggested that retaining $25 \%$ mTeSR1 medium facilitated the increased proportion of ALB-positive hepatocyte-like cells derived from $\mathrm{H} 1$ cells. 
Functional analysis
of hepatocyte-
like cells derived
from H1 cells via
the differentiation
method including
$25 \%$
medium
WerTeSR1 compared the functions of the $0 \%$ and $25 \%$ groups and found that the $25 \%$ group displayed the highest percentage of ALB-positive cells, while the $0 \%$ group displayed the highest rate as a control. Next, the ALB secretion activity was detected. The ALB secretion of the $25 \%$ group approached 2.334 $\pm 0.127 \mathrm{mg} / \mathrm{L}$, while that of the $0 \%$ group was $2.084 \pm 0.097 \mathrm{mg} / \mathrm{L}$ $(p=0.073$, Fig. 3A). Then, the urea production level was also detected in the media with an ELISA, and urea production levels were

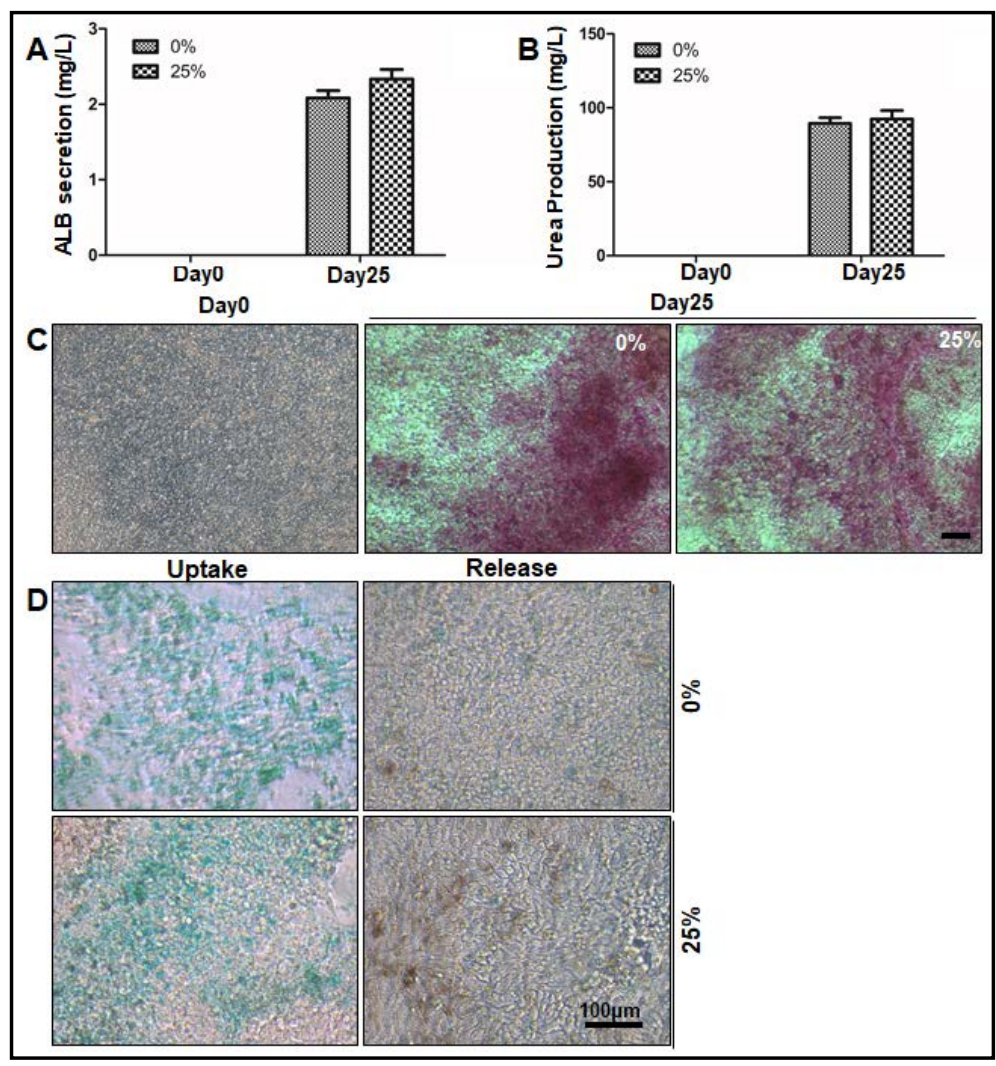

Fig. 3. Functional testing of the mature differentiated cells derived from H1 cells. Note: (A) ALB secretion of differentiated cells was detected by ELISA at day 25; (B) urea production by differentiated cells was detected by a urea assay kit on day 25; (C) ICG uptake and release; and (D) PAS staining of the differentiated cells was also detected at day 25 . $92.415 \pm 5.737 \mathrm{mg} / \mathrm{L}$ in the $25 \%$ group and $89.382 \pm 3.925 \mathrm{mg} / \mathrm{L}$ in the $0 \%$ group ( $\mathrm{p}=0.492$, Fig. 3B). Moreover, glycogen storage was detected by PAS staining in the differentiated cells. Glycogen content was increased significantly in the differentiated cells compared with the undifferentiated cells, but no significant difference was observed between the $25 \%$ and $0 \%$ groups (Fig. 3C). The ICG uptake and release of differentiated cells were also detected (Fig. 3D). The differentiated cells of both groups exhibited green staining, almost all of the cells released ICG after $12 \mathrm{~h}$ in complete medium with the withdrawal of ICG, and no significant difference was observed between the $25 \%$ and $0 \%$ groups. These results showed that similar functional activity of the hepatocyte-like cells was observed in the $25 \%$ and $0 \%$ groups.

The differentiation method retaining 25\% mTeSR1 medium could facilitate the generation of functional hepatocyte-like cells derived from iPS cells

To further confirm the novel hepatic differentiation method, we used an iPS cell line to generate hepatocyte-like cells with $25 \%$ mTeSR1 medium retained during differentiation. Fig. 4A shows the cell morphology changes during the differentiation of iPS cells. The differentiated cells displayed endoderm cellmorphology at day 4, early hepatic cellmorphology at day 15, and mature hepatocyte morphology at day 25. Further immunofluorescence results showed that the differentiated cells expressed the endoderm markers SOX17 and FOXA2 on day 4, the hepatic precursor markers AFP and HNF4 $\alpha$ on day 15, and the mature hepatocyte marker ALB on day 25 (Fig. 4B). The ALB secretion of the differentiated cells approached 1.82 $\pm 0.091 \mathrm{mg} / \mathrm{L}$ (Fig. 4C), and the urea production levels were $77.608 \pm 3.880 \mathrm{mg} / \mathrm{L}$ (Fig. 4D). Moreover, the differentiated cells also displayed glycogen storage ability (Fig. 4E) and ICG 
Fig.

Characterization of differentiated cells derived from iPS cells with $25 \%$ concentration of mTeSR1 medium during hepatic differentiation. Note: (A) The cells on days $0,4,15$ and 25 in bright-field microscopy; (B) the expression levels of SOX17 and FOXA2 at day $4, \mathrm{AFP}$ and HNF $4 \alpha$ at day 15 and ALB at day 25 were detected in differentiated cells by immunostaining; (C) ALB secretion by differentiated cells was detected by

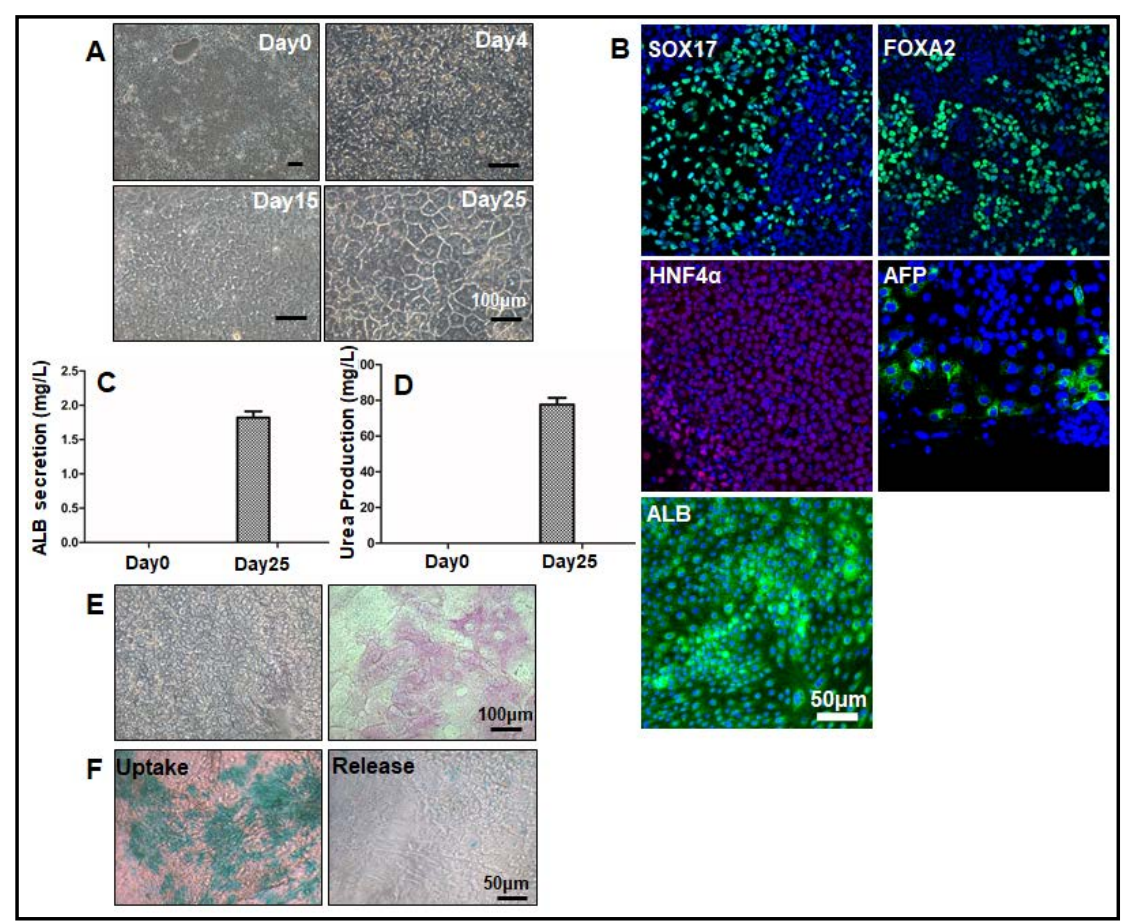
ELISA at day 25; (D) urea production by differentiated cells was detected by a urea assay kit on day 25; (E) PAS staining and (F) ICG uptake and release of the differentiated cells were also detected at day 25.

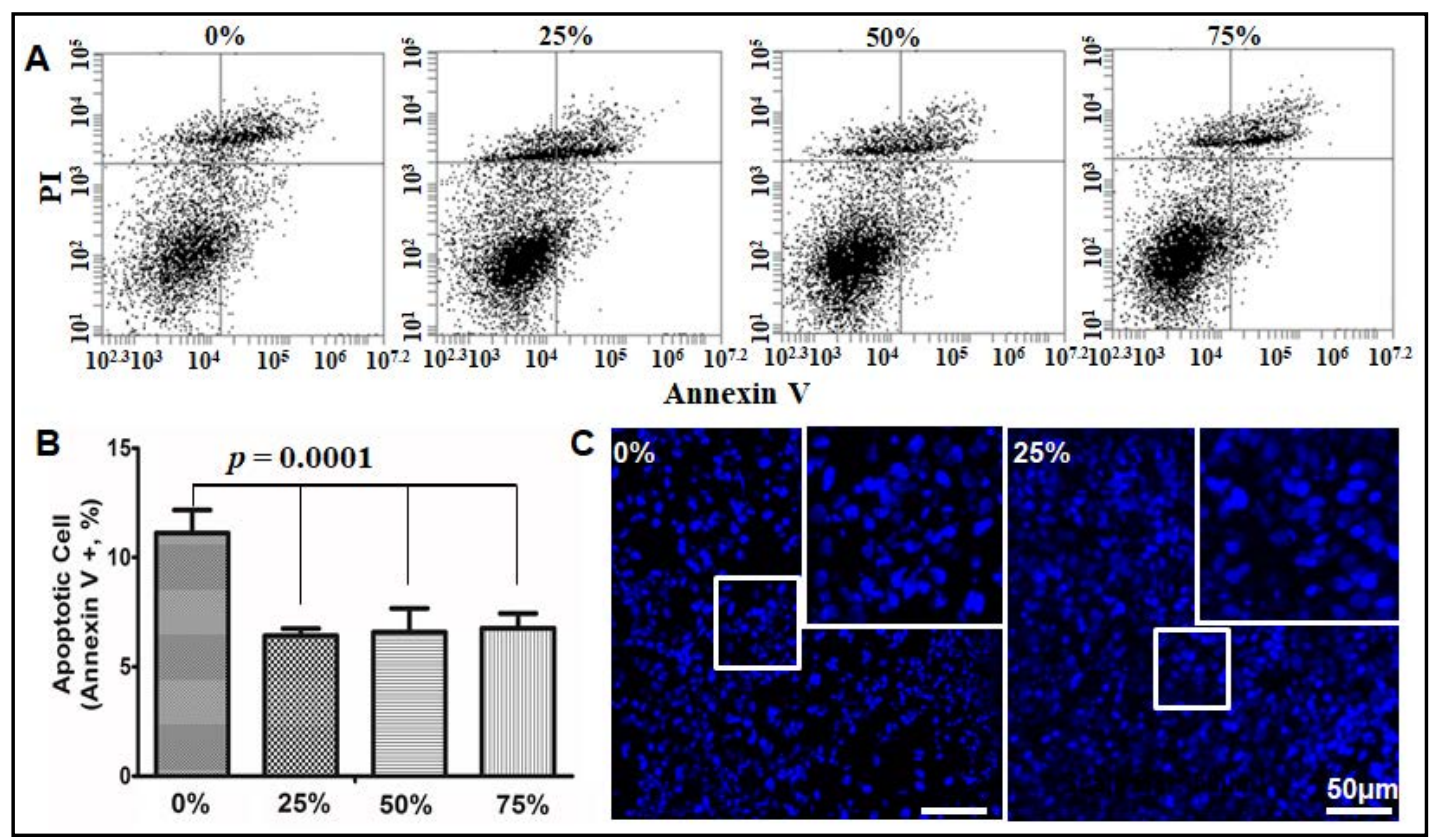

Fig. 5. Apoptosis was investigated in differentiated cells derived from H1 cells at day 25. Note: Flow cytometry analysis using Annexin V-FITC and propidium iodide (PI) double staining was performed to detect the apoptosis ratio. (A) the typical images; (B) statistic results of the apoptosis ratio ( $\mathrm{n}=3$ ); (C) cells stained with Hoechst 33258 were observed by fluorescence microscopy. 
uptake and release (Fig. 4F). These data suggested that the improved differentiation method, in which $25 \%$ of the mTeSR1 medium was retained, was appropriate for differentiating iPS cells into hepatocyte-like cells.

\section{Retaining 25\% of the mTeSR1 medium during hepatic differentiation could decrease cell apoptosis}

During hepatic differentiation of $\mathrm{H} 1$ cells, we observed that the cell morphology of the $25 \%$ mTeSR1 group was better and had fewer dead cells than the other groups. Thus, we hypothesized that retaining $25 \%$ of the mTeSR1 medium during hepatic differentiation might decrease cell apoptosis. To test this hypothesis, flow cytometry was used to quantify apoptosis in differentiated cells. At day 25, the differentiated cells with $0 \%, 25 \%, 50 \%$ or $75 \%$ mTeSR 1 were stained with the early apoptotic marker Annexin V-FITC. The populations of the Annexin V-FITC-positive cells were $11.12 \% \pm 1.05 \%$ in the $0 \%$ group, $6.43 \% \pm 0.32 \%$ in the $25 \%$ group, $6.58 \% \pm 1.09 \%$ in the $50 \%$ group, and $6.77 \% \pm 0.67 \%$ in the $75 \%$ group (Fig. 5A, 5B). Then, we mainly focused on the apoptotic nuclei morphology of the $0 \%$ and $25 \%$ groups using Hoechst 33258 staining. As shown in Fig. 5B, most cells in the $25 \%$ group had regular nuclei, with only a few showing apoptotic nuclei with condensed chromatin, while increased apoptotic nuclear morphology was shown in the $0 \%$ group. These results indicated that retaining $25 \%$ of the mTeSR 1 medium during hepatic differentiation could decrease cell apoptosis.

\section{Discussion}

The directed differentiation of human ES or iPS cells into hepatocytes could provide a renewable source of exogenous hepatocytes for drug toxicity testing and cell-based therapeutics [14]. However, the ability to use primary hepatocytes either for therapeutic purposes or basic research has been frustrated by their tendency to rapidly dedifferentiate and lose most hepatic functions after growth in a tissue culture environment [15]. The need to expand primary hepatocytes purified from donor liver tissue could be avoided by using ES and iPS cells for their indefinite proliferation without a loss of potency.

Some growth factors were reported to be important in hepatic differentiation. Activin A and BMP4 were used for definitive endoderm (DE) formation, which was the first and one of the most crucial steps in the hepatic differentiation of ES/iPS cells $[16,17]$. In our study, we also found high expression levels of SOX17 and FOXA2. For hepatic progenitor differentiation, BMP2, FGF, HGF, and KGF were used with high AFP and HNF4 $\alpha$ expression levels, while for hepatic maturation, oncostatin-M was used to induce ALB [18-24]. However, in this study, the number of dead cells was substantial within 3 days after differentiation, and the hepatocyte-like cells could not survive beyond 25 days. To improve the cell survival rate, we tried adding different concentrations of mTeSR1 medium to the stages of DE and hepatic progenitor differentiation. Interestingly, we found that fewer cells died during differentiation and that hepatocyte-like cells could survive well beyond day 35 in the mTeSR1 medium treatment groups. The expression levels of the DE markers and hepatic progenitor markers were slightly different between the $0 \%$ and $25 \%$ groups. However, the $25 \%$ mTeSR 1 group showed a higher proportion of ALB-positive cells than the other groups, which was generally consistent with a previous study [25]. Similar hepatocyte functional activities were found in the $0 \%$ and $25 \%$ mTeSR 1 groups. Moreover, iPS cells could also be differentiated into hepatocyte-like cells using this method with 25\% mTeSR 1 and could survive well beyond 40 days in our experiment. Evidence shows that culture with low serum concentrations could improve ICG uptake function as well as mature hepatic marker expression [26]. In the present study, we did not observe a significant difference in hepatic marker expression and function between the $0 \%$ and $25 \%$ mTeSR 1 groups. However, we found that retaining $25 \%$

\section{KARGER}


of the mTeSR1 medium during hepatic differentiation could decrease hepatocyte-like cell apoptosis. Taken together, these results suggest that retaining $25 \%$ of the mTeSR1 medium during hepatic differentiation could increase the proportion of ALB-positive cells and cell survival by decreasing cell apoptosis.

The components of the mTeSR1 medium is complicated, including the growth factors, vitamins, lipids, amino acids, trace minerals, inorganic salts et al. It is difficult for us to illuminate which component play a primary role. One of the components caused our attention. bFGF can synergize with Noggin, an antagonist of BMPs, to maintain human ES cell culture via inhibition of caspase activation $[27,28]$. bFGF and Activin A function to promote survival and proliferation of single iPS cells in conditioned half-exchange mTeSR1 medium [29]. Furthermore, acidic FGF (aFGF) and bFGF produced by the adjacent cardiac mesoderm could induce the hepatocyte lineage [22]. During this stage, BMPs produced by the transversum mesenchyme could promote the ability of endoderm to respond to FGFs [22]. bFGF is used for hepatic specification [30]. We also concern about the other components, TGF- $\beta 1$ and insulin. TGF- $\beta 1$ could induce apoptosis in liver, however, insulin could inhibit it [31-33], which maybe the apoptosis induced by TGF- $\beta 1$ could not be observed in our experiment. Thus, we speculated that the complicated mechanisms of the bFGF, BMPs and Activin A might exist to regulate the cells survival in definitive endoderm specification and to facilitate hepatic specification in our experiment, in which both the antagonism and synergism of the growth factors were involved.

There are also some limitations in this study. We did not investigate the specific components of mTeSR1 medium or the molecular factors involved in regulating hepatocytelike cell survival. Thus, we do not know whether retaining $25 \%$ of the mTeSR 1 medium is appropriate for all hepatic differentiation methods. Nonetheless, this report offers a clue for mTeSR1 medium culture conditions during hepatic differentiation, expanding our knowledge of hepatocyte induction in vitro.

\section{Acknowledgements}

The authors greatly appreciate the editors and the anonymous peer reviewers for their critical reading and insightful comments, which have improved our manuscript substantially. This study was supported by the National Key R\&D Program of China (2017YFC1105000), the National Natural Science Foundation of China (81770319, 81503170, 81570039, 81400307, and 81370215,), the Guangdong Provincial Natural Science Foundation (2017A030313757, 2016A030313170, and 2014A030310058), and the Sci-Tech Planning Project of Zhejiang Province (2015C33295 and 2015C37130).

\section{Disclosure Statement}

The authors have no conflicts of interest to disclose.

\section{References}

1 Amano H, Hino H, Tateno C, Emoto K, Imaoka Y, Yamasaki C, Itamoto T, Tashiro H, Asahara T, Ohdan H, Yoshizato K: Therapeutic potential of propagated hepatocyte transplantation in liver failure. J Surg Res 2011;167:e29-37.

2 Gupta S, Chowdhury JR: Therapeutic potential of hepatocyte transplantation. Semin Cell Dev Biol 2002;13:439-446.

3 Enns GM, Millan MT: Cell-based therapies for metabolic liver disease. Mol Genet Metab 2008;95:3-10.

4 Clayton DF, Darnell JE, Jr: Changes in liver-specific compared to common gene transcription during primary culture of mouse hepatocytes. Mol Cell Biol 1983;3:1552-1561. 


\section{Cellular Physiology Cell Physiol Biochem 2018;51:1533-1543 and Biachemistry \begin{tabular}{l|l} 
DOI: 10.1159/000495644 2018 The Author(s). Published by S. Karger AG, Basel \\
Publis
\end{tabular}

Hou et al.: mTeSR1 Medium during Hepatic Differentiation

5 Ma X, Duan Y, Tschudy-Seney B, Roll G, Behbahan IS, Ahuja TP, Tolstikov V, Wang C, McGee J, Khoobyari S, Nolta JA, Willenbring H, Zern MA: Highly efficient differentiation of functional hepatocytes from human induced pluripotent stem cells. Stem Cells Transl Med 2013;2:409-419.

6 Zheng Y, Zhou J, Li X, Xu G, Jin M, Shen R, Su R, Zhan S, Ding B, Jia M, Cui Y, Yu X: Mir-382 Promotes Differentiation of Rat Liver Progenitor Cell WB-F344 by Targeting Ezh2. Cell Physiol Biochem 2018;48:2389-2398.

-7 Song Z, Cai J, Liu Y, Zhao D, Yong J, Duo S, Song X, Guo Y, Zhao Y, Qin H, Yin X, Wu C, Che J, Lu S, Ding M, Deng H: Efficient generation of hepatocyte-like cells from human induced pluripotent stem cells. Cell Res 2009;19:1233-1242.

-8 Sullivan GJ, Hay DC, Park IH, Fletcher J, Hannoun Z, Payne CM, Dalgetty D, Black JR, Ross JA, Samuel K, Wang G, Daley GQ, Lee JH, Church GM, Forbes SJ, Iredale JP, Wilmut I: Generation of functional human hepatic endoderm from human induced pluripotent stem cells. Hepatology 2010;51:329-335.

-9 Keller G: Embryonic stem cell differentiation: emergence of a new era in biology and medicine. Genes Dev 2005;19:1129-1155.

10 Xue Y, Cai X, Wang L, Liao B, Zhang H, Shan Y, Chen Q Zhou T, Li X, Hou J, Chen S, Luo R, Qin D, Pei D, Pan G: Generating a non-integrating human induced pluripotent stem cell bank from urine-derived cells. PloS one 2013;8:e70573.

11 Zhang S, Chen S, Li W, Guo X, Zhao P, Xu J, Chen Y, Pan Q, Liu X, Zychlinski D, Lu H, Tortorella MD, Schambach A, Wang Y, Pei D, Esteban MA: Rescue of ATP7B function in hepatocyte-like cells from Wilson's disease induced pluripotent stem cells using gene therapy or the chaperone drug curcumin. Human molecular genetics 2011;20:3176-3187.

12 Hu B, Wu T, Zhao Y, Xu G, Shen R, Chen G: Physiological Signatures of Dual Embryonic Origins in Mouse Skull Vault. Cell Physiol Biochem 2017;43:2525-2534.

-13 Liu J, Zhu Y, Chen S, Shen B, Yu F, Zhang Y, Shen R: Apocynin Attenuates Cobalt Chloride-Induced Pheochromocytoma Cell Apoptosis by Inhibiting P38-MAPK/Caspase-3 Pathway. Cell Physiol Biochem 2018;48:208-214.

14 Grompe M: Principles of therapeutic liver repopulation. J Inherit Metab Dis 2006;29:421-425.

15 Strain AJ: Isolated hepatocytes: use in experimental and clinical hepatology. Gut 1994;35:433-436.

16 Kubo A, Shinozaki K, Shannon JM, Kouskoff V, Kennedy M, Woo S, Fehling HJ, Keller G: Development of definitive endoderm from embryonic stem cells in culture. Development 2004;131:1651-1662.

17 Gouon-Evans V, Boussemart L, Gadue P, Nierhoff D, Koehler CI, Kubo A, Shafritz DA, Keller G: BMP-4 is required for hepatic specification of mouse embryonic stem cell-derived definitive endoderm. Nature biotechnology 2006;24:1402-1411.

18 Shirahashi H, Wu J, Yamamoto N, Catana A, Wege H, Wager B, Okita K, Zern MA: Differentiation of human and mouse embryonic stem cells along a hepatocyte lineage. Cell transplantation 2004;13:197-211.

19 Cai J, Zhao Y, Liu Y, Ye F, Song Z, Qin H, Meng S, Chen Y, Zhou R, Song X, Guo Y, Ding M, Deng H: Directed differentiation of human embryonic stem cells into functional hepatic cells. Hepatology 2007;45:12291239.

20 Hay DC, Zhao D, Fletcher J, Hewitt ZA, McLean D, Urruticoechea-Uriguen A, Black JR, Elcombe C, Ross JA, Wolf R, Cui W: Efficient differentiation of hepatocytes from human embryonic stem cells exhibiting markers recapitulating liver development in vivo. Stem cells 2008;26:894-902.

-21 Agarwal S, Holton KL, Lanza R: Efficient differentiation of functional hepatocytes from human embryonic stem cells. Stem cells 2008;26:1117-1127.

22 Zaret KS: Hepatocyte differentiation: from the endoderm and beyond. Current opinion in genetics \& development 2001;11:568-574.

23 Zaret KS, Grompe M: Generation and regeneration of cells of the liver and pancreas. Science 2008;322:1490-1494.

24 Drummond R: Does a structural bridge exist between the DNA and the specialized cytoplasmic organelles during the early part of their development? A mechanism for the positioning of flagella and possibly other cytoplasmic organelles. J Theor Biol 2003;223:309-312.

25 Si-Tayeb K, Noto FK, Nagaoka M, Li J, Battle MA, Duris C, North PE, Dalton S, Duncan SA: Highly efficient generation of human hepatocyte-like cells from induced pluripotent stem cells. Hepatology 2010;51:297305. 


\section{Cellular Physiology Cell Physiol Biochem 2018;51:1533-1543 and Biochemistry $\begin{gathered}\text { DOl: 10.1159/000495644 } \\ \text { Published }\end{gathered}$

Hou et al.: mTeSR1 Medium during Hepatic Differentiation

26 He Y, Zhang WY, Gong M, Huang JY, Tang N, Feng T, Wei GH, He TC, Bi Y: Low serum concentration facilitates the differentiation of hepatic progenitor cells. Saudi Med J 2011;32:128-134.

27 Xu RH, Peck RM, Li DS, Feng X, Ludwig T, Thomson JA: Basic FGF and suppression of BMP signaling sustain undifferentiated proliferation of human ES cells. Nat Methods 2005;2:185-190.

-28 Wang X, Lin G, Martins-Taylor K, Zeng H, Xu RH: Inhibition of caspase-mediated anoikis is critical for basic fibroblast growth factor-sustained culture of human pluripotent stem cells. J Biol Chem 2009;284:3405434064.

29 Guo X, Lian R, Guo Y, Liu Q, Ji Q, Chen J: bFGF and Activin A function to promote survival and proliferation of single iPS cells in conditioned half-exchange mTeSR1 medium. Hum Cell 2015;28:122-132.

-30 Anzalone R, Lo Iacono M, Corrao S, Magno F, Loria T, Cappello F, Zummo G, Farina F, La Rocca G: New emerging potentials for human Wharton's jelly mesenchymal stem cells: immunological features and hepatocyte-like differentiative capacity. Stem Cells Dev 2010;19:423-438.

-31 Oberhammer F, Fritsch G, Pavelka M, Froschl G, Tiefenbacher R, Purchio T, Schulte-Hermann R: Induction of apoptosis in cultured hepatocytes and in the regressing liver by transforming growth factor-beta 1 occurs without activation of an endonuclease. Toxicol Lett 1992;64-65 Spec No:701-704.

-32 Chen RH, Chang TY: Involvement of caspase family proteases in transforming growth factor-beta-induced apoptosis. Cell Growth Differ 1997;8:821-827.

-33 Chen RH, Su YH, Chuang RL, Chang TY: Suppression of transforming growth factor-beta-induced apoptosis through a phosphatidylinositol 3-kinase/Akt-dependent pathway. Oncogene 1998;17:1959-1968. 